\title{
Element abundance ratios in stellar population modelling
}

\author{
Daniel Thomas \\ Institute of Cosmology and Gravitation, University of Portsmouth, Dennis Sciama Building, \\ Burnaby Road, Portsmouth, PO1 3FX, UK. email: daniel.thomas@port.ac.uk \\ SEPnet, South East Physics Network, (www.sepnet.ac.uk)
}

\begin{abstract}
I review the implementation of the effects from varying chemical element abundance ratios in stellar population modelling, focusing on $\alpha$ - and Fe-peak elements. A brief overview of the development of such models over the past 30 years is provided, starting with early work on the identification of relevant absorption features in the spectra of early-type galaxies in the 1980s leading to the most recent developments of the past years. Recent highlights include the adoption of new flux calibrated libraries, the inclusion of a wide range of chemical elements, the calculation of error estimates on the model, and the consideration of element variation effects on full spectra. The calibration of such models with globular clusters and some key results on the element ratios measured in early-type galaxies are presented.
\end{abstract}

Keywords. galaxies: elliptical and lenticular, cD - galaxies: evolution - galaxies: formation

\section{Introduction}

The spectra of galaxies and globular clusters carry a wealth of information about gas and stellar population properties. Emission lines are used to derive gas kinematics, star formation activity and black hole accretion (e.g. Kauffmann et al. 2003; Tremonti et al. 2004; Sarzi et al. 2006; Schawinski et al. 2007; Thomas et al. 2013), the absorption line and stellar continuum component of the spectrum, instead, discloses stellar population properties that act as fossil record and can be used to derive ages, formation epochs, star formation histories, and element abundances (e.g. Trager et al. 2000a; Kuntschner 2000; Thomas et al. 2005; Nelan et al. 2005; Thomas et al. 2010). The absorption features in a spectrum are particularly useful for several reasons. They can easily be measured and calibrated to a common system (Burstein et al. 1984; Faber et al. 1985), they are largely insensitive to dust attenuation (MacArthur 2005), and they allow to dissect 'metallicity' into individual element abundances (Greggio 1997; Tantalo et al. 1998; Trager et al. 2000b; Thomas et al. 2003a) that in turn set valuable constraints on the chemical enrichment history (Thomas et al. 1999). The inclusion of the these effects in stellar modelling has produced a major step forward in the field of stellar population modelling and has led to key progress in the interpretation of galaxy data.

\section{Brief history of element ratio modelling}

In the 1980s the Lick group have defined optical absorption-line indices (Burstein et al. 1984; Faber et al. 1985; Gorgas et al. 1993) that are by far the most commonly used in absorption-line analyses of old stellar populations. The bandpasses of Lick indices are relatively large with widths up to $50 \AA$ and two windows blue- and redward of the band-pass defining pseudo-continua. They have been designed for massive galaxies with significant line broadening through random motions of the stars. The advantage is that a 


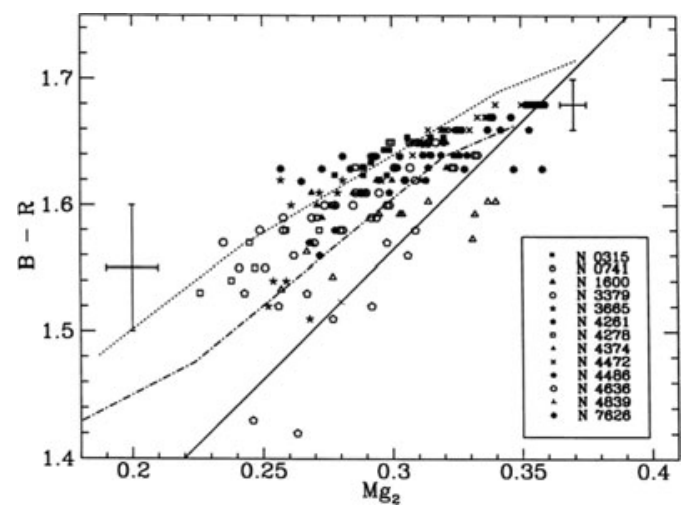

Figure 1. Measurements of $\mathrm{Mg}$ vs Fe absorption line indices for early-type galaxies confronted with stellar population models. From Davies et al.(1993).

wide bandpass increases the signal-to-noise ratio, and measurements are relatively robust. The downside is that they are an agglomeration of a large number of absorption lines from various chemical elements, so that their use for the derivation of individual element abundances is nontrivial.

While the 1990s have seen the advent of a number of stellar population models produced for the aim of analysing galaxy spectra (Buzzoni 1989; Bruzual \& Charlot 1993; Vazdekis et al. 1996; Worthey 1994; Maraston 1998), it was also recognised that Mg and Fe absorption features in early-type galaxies could not be reproduced by these models (Peletier 1989; Worthey et al. 1992; Davies et al. 1993; Carollo \& Danziger 1994; Bender \& Paquet 1995; Greggio 1997) as shown in Fig. 1. Tripicco \& Bell (1995) made a critical step forward and determined the sensitivity of Lick absorption-line indices to individual element abundance variations through model atmosphere calculations.

The first stellar population models with variable abundance element ratios using these calculations were then generated by Thomas et al. (2003a) based on a method developed by Tantalo et al. (1998) and Trager et al. (2000b). A key in the development of these models was the empirical calibration with globular cluster data by Maraston et al. (2003). The models have subsequently been updated with new model atmosphere calculations by Korn et al. (2005, see also Houdashelt et al.2005) for non-solar metallicities and the higher-order Balmer line indices. The latter turned out to be key for the interpretation of bluer Balmer absorption strengths in galaxies. Because of the presence of significant Fe absorption in the pseudo continua of the Balmer indices $\mathrm{H} \gamma$ and $\mathrm{H} \delta$, both features are highly sensitive to $\alpha / \mathrm{Fe}$ ratios leading to relatively strong index strengths in old, metal-rich stellar populations (Thomas et al. 2004). The inclusion of this effect is critical for the correct derivation of galaxy ages from these features (Thomas \& Davies 2006).

More recently, a number of other element abundance sensitive models have been produced increasing the number of chemical elements considered, implementing other stellar libraries, and extending the approach to full spectral response (Annibali et al. 2007; Schiavon 2007; Coelho et al. 2007; Lee et al. 2009; Thomas et al. 2011; Worthey et al. 2011; Conroy et al. 2014).

\section{Recent progress}

In the following I will discuss in more detail some of the recent developments. 


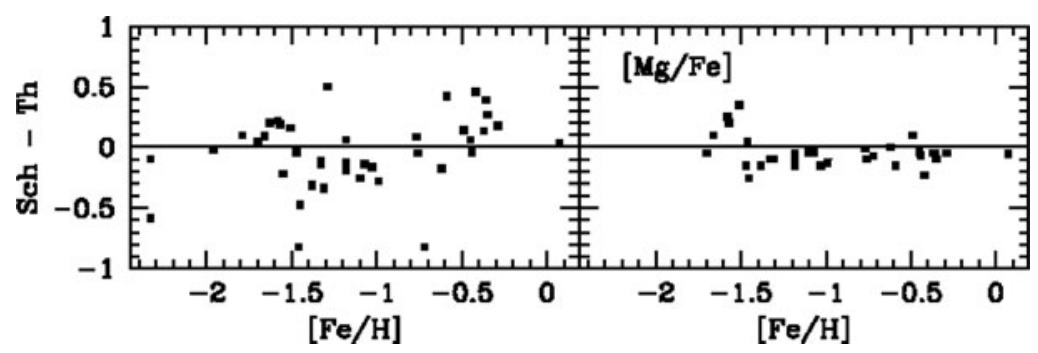

Figure 2. Globular cluster element abundances $[\mathrm{Fe} / \mathrm{H}]$ and $[\mathrm{Mg} / \mathrm{Fe}]$ from Thomas et al. (2011) and Schiavon (2007) models. R. Schiavon (private communication).
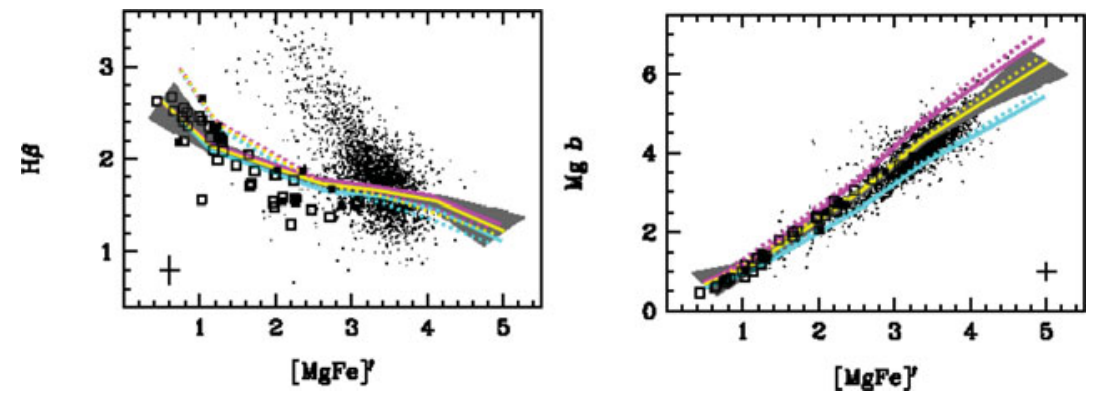

Figure 3. Calibration of the $\mathrm{H} \beta$ and $\mathrm{Mg} b$ indices with galactic globular clusters (squares). Models with an age of $13 \mathrm{Gyr}$ and various metallicities and $\alpha / \mathrm{Fe}$ ratios are shown. The grey shaded area along the model with $\alpha / \mathrm{Fe}]=0.3$ dex (yellow line) indicates the $1-\sigma$ error of the model prediction. The small black dots are SDSS early-type galaxies from Thomas et al. (2010). From Thomas et al. (2011).

\subsection{New stellar libraries}

The advent of new flux-calibrated libraries has made it possible to produce models that are now flux calibrated and at higher spectral resolution, and in particular not tied anymore to the Lick/IDS system. This is essential for the interpretation of galaxy spectra where calibration stars are not available, such as large galaxy redshift surveys or other high-redshift observations. Johansson et al. (2010) produced new empirical fitting functions based on the MILES library (Sánchez-Blázquez et al. 2006) that relate absorption line strength with stellar parameters, that are adopted in the Thomas et al. (2011) models. These models can directly be applied to SDSS data with minimal correction for spectral resolution (Beifiori et al. 2011).

The models have been calibrated with galactic globular clusters (Puzia et al. 2002; Schiavon et al. 2005) in Thomas et al. (2011). Ages and total metallicities and some element ratios are well reproduced, while some element ratios remain uncertain. It is encouraging, though, that different models give consistent result for some element ratios as shown in Fig. 2 presenting the comparison of globular cluster element abundances $[\mathrm{Fe} / \mathrm{H}]$ and $[\mathrm{Mg} / \mathrm{Fe}]$ from Thomas et al. (2011) and Schiavon (2007) models.

\subsection{Inclusion of model errors}

A particular feature of the Thomas et al. (2011) models is that model errors are calculated for the first time. This is possible thanks to the use of absorption line strengths in combination with fitting functions. The major strength of fitting functions lies in the fact that they allow for interpolation between well-populated regions of stellar parameter space which increases the accuracy of the model in stellar parameter space that is only sparsely sampled by empirical stellar libraries. Moreover, each absorption index or spec- 

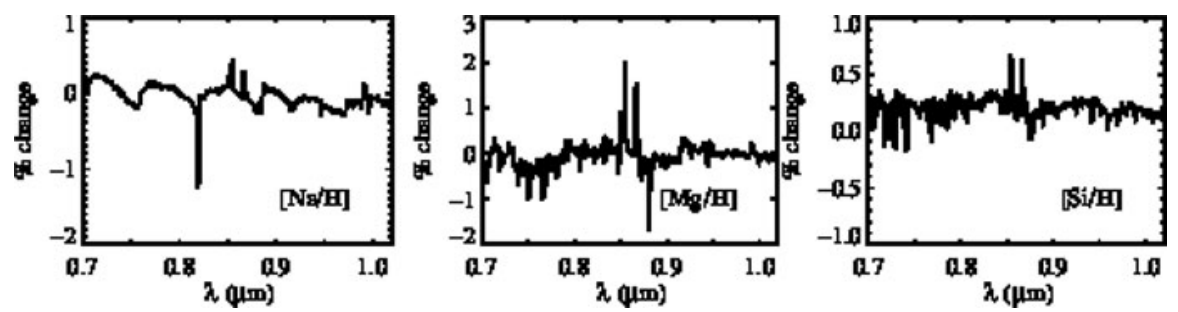

Figure 4. An example of the wavelength dependent response to the variation of the elements $\mathrm{Na}, \mathrm{Mg}$, and Si. From Conroy et al. (2014).

tral feature is represented by an individual fitting function, which is optimised to best reproduce its behaviour in stellar parameter space.

The construction of the model through fitting functions allows to make a straightforward assessment of the statistical errors on each individual index prediction that is caused by the uncertainties in the index calibrations. Thomas et al. (2011) calculate errors in the model predictions through Monte Carlo simulations. Both index measurements and stellar parameters for each star are perturbed in 600 realisations per simulation using the errors given in Sánchez-Blázquez et al. (2006). For each realisation they re-derive the fitting functions and insert those in the the stellar population code. This yields 600 index predictions per index and stellar population parameter age, metallicity, and $\alpha / \mathrm{Fe}$ ratio. They then fit a Gaussian to the distribution of index strengths and derive the 1- $\sigma$ error. Note that these errors do not include systematic effects such as a change of stellar evolutionary track, for instance.

Fig. 3 illustrates the effect. Model errors are generally very small and well below the observational errors for globular clusters or galaxies around solar metallicity. Model errors rise considerably and become comparable to the typical observational error toward the highest and lowest metallicities. This behaviour ought to be expected and is a direct consequence of the empirical stellar library, in which the stellar parameter space is inevitably sampled worse at the ends of the distribution. As a consequence, model errors turn out to be quite large at the edges. An increase of sampling density in stellar libraries would therefore be highly beneficial in these sections of the parameter space.

\subsection{Full spectral response}

An alternative to the approach of Thomas et al. (2003a) has been presented in the recent literature. Conroy et al. (2014) follow the method of Thomas et al. (2003a) in that they use response functions from model atmosphere calculations to assess the relative effect of element abundance variations, while the basic stellar population model is footed on empirical stellar libraries. Rather than using response functions for key absorption features, though, they calculate wavelength-dependent spectral response functions and apply those to the full model spectrum. This approach is very powerful but somewhat more affected by uncertainties in line lists and continuum uncertainties, hence may be considered well complementary to the use of absorption index features. An example of the wavelength dependent response to the variation of the elements $\mathrm{Na}, \mathrm{Mg}$, and $\mathrm{Si}$ is shown in Fig. 4.

\subsection{Galaxy data}

Element ratio sensitive stellar population models provide the opportunity to derive the abundance ratios for a variety of chemical elements of unresolved stellar populations. Graves \& Schiavon (2008) and Johansson et al. (2012) developed codes that fit iteratively a set of absorption line indices resulting in the derivation of the abundance ratios of the 

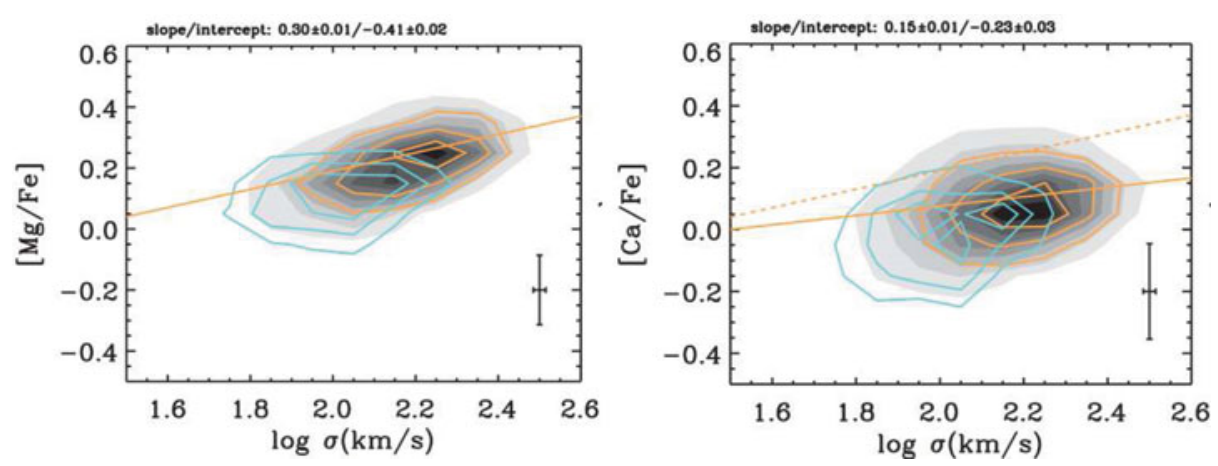

Figure 5. Element ratios $[\mathrm{Mg} / \mathrm{Fe}]$ and $[\mathrm{Ca} / \mathrm{Fe}]$ as a functions of velocity dispersion in early-type galaxies. The solid line is a linear fit, the dotted line shows the correlation for $[\mathrm{Mg} / \mathrm{Fe}]$. From Johansson et al. (2012).

elements best accessible through strong absorption features including $\mathrm{C}, \mathrm{N}, \mathrm{Mg}, \mathrm{Ca}$, Ti, and Fe. The abundance of oxygen cannot be derived directly from absorption features, but can be estimated through its role as dominating element among the light $\alpha$-elements (Johansson et al. 2012). Conroy et al. (2014) recently extended this procedure to full spectral fits adding the element Si.

A key result seen in all these studies consistently is that the light-element to Fe-peak abundance ratios $[\mathrm{C} / \mathrm{Fe}],[\mathrm{N} / \mathrm{Fe}],[\mathrm{O} / \mathrm{Fe}],[\mathrm{Mg} / \mathrm{Fe}]$, and $[\mathrm{Si} / \mathrm{Fe}]$ increase with galaxy velocity dispersion and mass (Smith et al. 2009; Johansson et al. 2012; Conroy et al. 2014), leading to significantly super-solar ratios in massive galaxies. The heavier elements Ca and $\mathrm{Ti}$ deviate from this trend. $[\mathrm{Ca} / \mathrm{Fe}]$ is close to solar across all galaxy masses and may only increase slightly (if at all) with galaxy mass (see Fig. 5). This has also been interpreted as Calcium under-abundance in massive galaxies (Thomas et al. 2003b). The best explanation is that the relevance of Type Ia enrichment increases with element mass, which implies that $\mathrm{Ca}$ and $\mathrm{Ti}$ are suppressed because they get partially enriched by the delayed Type Ia supernovae. However, Ti, even though being heavier than Ca, appears to show somewhat higher enhancement than $\mathrm{Ca}$ in massive galaxies, which seems contrived within this picture. The optical spectrum used in these studies only is moderately sensitive to $\mathrm{Ti}$ abundance, though, and further work on this, potentially including the near-IR part of the spectrum, will be very valuable.

\section{References}

Annibali, F., Bressan, A., Rampazzo, R., Zeilinger, W. W., \& Danese, L., 2007, A\&A, 463, 455

Beifiori, A., Maraston, C., Thomas, D., \& Johansson, J., 2011, A\&\&A, 531, 109

Bender, R. \& Paquet, A., 1995, in van der Kruit P. C., Gillmore G., eds, Stellar Populations IAU Symposium 164. Kluwer Academic Publishers, Dordrecht, p. 259

Bruzual, A. G. \& Charlot S., 1993, ApJ, 405, 538

Burstein, D., Faber, S. M., Gaskell, C. M., \& Krumm, N., 1984, ApJ, 287, 586

Buzzoni, A., 1989, ApJS, 71, 817

Carollo, C. M. \& Danziger, I. J., 1994, MNRAS, 270, 523

Coelho, P., Bruzual, G., Charlot, S., Weiss, A., Barbuy, B., \& Ferguson, J. W., 2007, MNRAS, 382,498

Conroy, C., Graves, G. J., \& van Dokkum, P. G., 2014, ApJ, 780, 33

Davies, R. L., Sadler, E. M., \& Peletier, R. F., 1993, MNRAS, 262, 650

Faber, S. M., Friel, E. D., Burstein, D., \& Gaskell, D. M., 1985, ApJS, 57, 711 
Gorgas, J., Faber, S. M., Burstein, D., González, J. J., Courteau, S., \& Prosser, C., 1993, ApJS, 86,153

Graves, G. J. \& Schiavon, R. P., 2008, ApJS, 177, 446

Greggio, L., 1997, MNRAS, 285, 151

Houdashelt, M. L., Trager, S. C., \& Worthey, G., 2005, Highlights of Astronomy, 13, 585

Johansson, J., Thomas, D., \& Maraston, C., 2010, MNRAS, 406, 165

Johansson, J., Thomas, D., \& Maraston, C., 2012, MNRAS, 421, 1908

Kauffmann, G., et al., 2003, MNRAS, 341, 33

Korn, A., Maraston, C., \& Thomas, D., 2005, A\& $A, 438,685$

Kuntschner, H., 2000, MNRAS, 315, 184

Lee, H., Worthey, G., Dotter, A., Chaboyer, B., Jevremović, D., Baron, E., Briley, M. M., Ferguson, J. W., Coelho, P., \& Trager, S. C., 2009, ApJ, 694, 902

MacArthur, L. A., 2005, ApJ, 623, 795

Maraston, C., 1998, MNRAS, 300, 872

Maraston, C., Greggio, L., Renzini, A., Ortolani, S., Saglia, R. P., Puzia, T., \& Kissler-Patig, M., 2003, $A \mathscr{E} A, 400,823$

Nelan, J. E., et al., 2005, ApJ, 632, 137

Peletier, R., 1989, Phd thesis, Rijksuniversiteit Groningen

Puzia, T., Saglia, R. P., Kissler-Patig, M., Maraston, C., Greggio, L., Renzini, A., \& Ortolani, S., 2002, A\&BA, 395, 45

Sánchez-Blázquez, P., Peletier, R. F., Jiménez-Vicente, J., Cardiel, N., Cenarro, A. J., FalcónBarroso, J., Gorgas, J., Selam, S., \& Vazdekis, A., 2006, MNRAS, 371, 703

Sarzi, M., Falcón-Barroso, J., Davies, R. L., Bacon, R., Bureau, M., Cappellari, M., de Zeeuw, P. T., Emsellem, E., Fathi, K., Krajnović, D., Kuntschner, H., McDermid, R. M., \& Peletier, R. F., 2006, MNRAS, 366, 1151

Schawinski, K., Thomas, D., Sarzi, M., Maraston, C., Kaviraj, S., Joo, S.-J., Yi, S. K., \& Silk, J., 2007, MNRAS, 382, 1415

Schiavon, R. P., 2007, ApJS, 171, 146

Schiavon, R. P., Rose, J. A., Courteau, S., \& MacArthur, L. A., 2005, ApJS, 160, 163

Smith, R. J., Lucey, J. R., Hudson, M. J., \& Bridges, T. J., 2009, MNRAS, 398, 119

Tantalo, R., Chiosi, C., \& Bressan, A., 1998, A\&A, 333, 419

Thomas, D. \& Davies, R. L., 2006, MNRAS, 366, 510

Thomas, D., Greggio, L., \& Bender, R., 1999, MNRAS, 302, 537

Thomas, D., Johansson, J., \& Maraston, C., 2011, MNRAS, 412, 2199

Thomas, D., Maraston, C., \& Bender, R., 2003a, MNRAS, 339, 897

Thomas, D., Maraston, C., \& Bender, R., 2003b, MNRAS, 343, 279

Thomas, D., Maraston, C., Bender, R., \& Mendes de Oliveira, C., 2005, ApJ, 621, 673

Thomas, D., Maraston, C., \& Johansson, J., 2011, MNRAS, 412, 2183

Thomas, D., Maraston, C., \& Korn, A., 2004, MNRAS, 351, L19

Thomas, D., Maraston, C., Schawinski, K., Sarzi, M., \& Silk, J., 2010, MNRAS, 404, 1775

Thomas, D., Steele, O., Maraston, C., Johansson, J., et al., 2013, MNRAS, 431, 1383

Trager, S. C., Faber, S. M., Worthey, G., \& González, J. J., 2000a, AJ, 120, 165

Trager, S. C., Faber, S. M., Worthey, G., \& González, J. J., 2000b, AJ, 119, 164

Tremonti, C. A., Heckman, T. M., Kauffmann, G., et al., 2004, ApJ, 613, 898

Tripicco, M. J. \& Bell, R. A., 1995, AJ, 110, 3035

Vazdekis, A., Casuso, E., Peletier, R. F., \& Beckmann, J. E., 1996, ApJS, 106, 307

Worthey, G., 1994, ApJS, 95, 107

Worthey, G., Faber, S. M., \& González, J. J., 1992, ApJ, 398, 69

Worthey, G., Ingermann, B. A., \& Serven, J., 2011, ApJ, 729, 148 